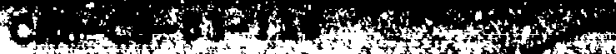

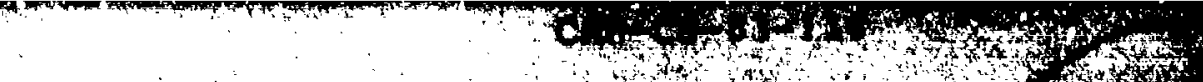

it

\title{
Metaphor \\ and Common-Sense Reasoning
}

Jaime G Carbonell and Steven Mititon

5 March 1883

DEPARTMENT

of

COMPUTER SCIENCE

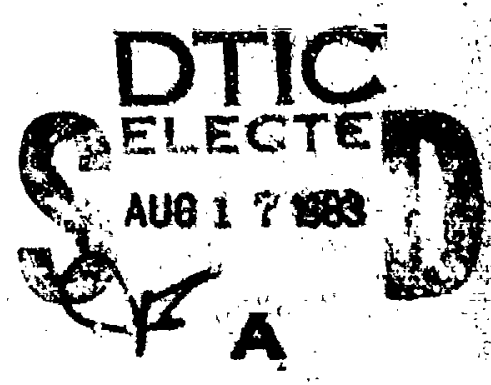

8
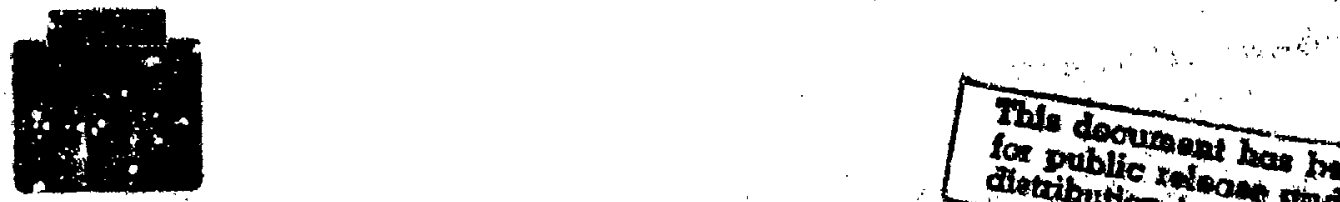

for pablicent ba bue

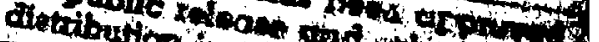

4

10
+4

Carnegre-Melton Urumersuty

$8308 \quad 16 \quad 005$ 


\title{
Metaphor and Common-Sense Reasoning
}

\author{
Jaime G Carbonell and Steven Minton \\ Carnegie-Mellon University \\ Pittsburgh, PA 15213
}

5 March 1983

\begin{abstract}
interenccs based on metaphors appear to play a major role in human common sense reasoning. This paper identifies and analyzes general inference patterns based upon ur Jerlying metaphors; in particular the pervasive balance principle. Strategies for metapnor comprehension are explored, and analogical mapping structures are proposed as a means of representing metaphorical relationships between domains. In addition, a framevisk for a computational model embodying principles of metaphorical common sense reasoning is discussed ta

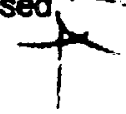

$-\mathrm{A}-$

\footnotetext{
${ }^{1}$ This research was sponfored in part by the Office of Nava! Research (ONA) under grant numbers NOOO14.79.C.OBe1 and No00 i4.82.C.50767.
} 
1. Introduction

\section{Table of Contents}

2. Experiential Reasoning vs Formal Systems

3. Patterns of Metaphorical Inference

3.1. The Balance Principle

3.2. The Physical Metaphor Hypothesis

4. The Role of Metaphor in Common.Sense Reasoning

5. Metaphorical Interence and The Mapping Problem

5.1. Knowledge Acquisition via Analogical Mappings

5.2. Salience and Novel Metaphors

5.3. A Classification Based on Processing Requirements

6. Representing Metaphors: The LIKE Relation

7. Generalizing Mapping Structures

8. Towards a Computational Model cf Metaphorical Inference

9. Conclusions

10. Bibliography 


\title{
Metaphor and Common Sense Reasoning
}

\author{
Jaime G. Carbonell and Steven Minton
}

\section{Introduction}

The theory that metaphor dominates large aspects of human thinking, as well playing a significant role in linguistic commur.ication, has been argued with considerable force $[26,24,8,5]$. However, the validity of such a theory is a matter of continuing debate that appears neither to dissuade its proponents nor convince its detractors. Being among the proponents, we propose to develop a computational reasoning system for performing metaphorical inferences. If such a system exhibits cognitively plausible common sense reasoning capabilities, it will demonstrate, at the very least, the utility of metaphorical inference in nodeling significant aspects of naive human reasoning. This paper reviews our initial steps towards the development of a computational model of metaphor-based reasoning.

\section{Experiential Reasoning vs Formal Systems}

Humans reason and learn from experience to a degree that no formal system, Al model, or philosophical theory has yet been able to explain. The statement that the human mind is (or contains) the sum total of its experiences is in itself rather vacuous. A more precise formulation of experiencebas. id reasoning must be structured in terms of coordinated answers to the following questions: How are experiences brought to bear in understanding new situations? How is long term' memory modified and indexed? How are inference patterns acquired in a particular domain and adapted to apply in novel situations? How does a person "see the light" when a previously incomprehensible problem is viewed from a new perspective? How are the vast majority of irrelevant or inappropriate experiences and inference patterns filtered out in the understanding process? Answering all these "how" questions requires a process model capable of organizing large amounts of knowledge and mapping relevant aspects of past experience to new situations. Some meaningful starts have been made towards large.scale episodic.based memory organization $[32,33,34,28,25]$ and towards episodic.based analogical reasoning $[9,12,7]$. Bearing these questions in mind, we examine the issue of common sense reasoning in knowledge-rich mundane domains.

Our central hypothesis is:

Experiential reasoning hypothesis: Reasoning in mundane, experience-rich recurrent situations is qualitatively different from formai, deductive reasoning evident in more abstract, experimentally contrived, or otherwise non-recurrent situations (such as some mathematical or puzzle-solving domains). 
In the statement of our hypothesis we do not mean to exclude experience-rich metaphorical inference from scientific or mathematical thought. Rather, we claim that formal deductive inference is definitely not the dominant process in mundane reasoning. In essence, the experiential reasoning hypothesis states that structuring new information according to relevant past experience is an important aspect of human comprehension .. ferhaps more important than other aspects studied thus far in much greater depth.

Common-sense experience-rich reasoning consists of recalling appropriate past experiences and inference patterns, whereas solving abstract problems divorced frnm real-world experience requires knowledge-poor search processes more typical of past and present Al problem solving systems. Since computer programs perform much better in simple, elegant, abstract domains than in "scruffy" experience-rich human domains, it is evident that a fundamental reasoning mechanism is lacking from the $\mathrm{Al}$ repertoire. The issue is not merely that $\mathrm{Al}$ systems lack experience in mundane human scenarios .. they would be unable to benefit from such experience if it were encoded in their knowledge base. We postulate that the missing reasoning method is based on the transfer of proven inference patterns and experiential knowledge across domains. This is not to say that humans are incapable of more formal reasoning, but rather that such reasoning is seldom necessary, and when applied it requires a more concerted cognitive effort than mundane metaphurical inference.

There is evidence that human expertise, far beyond what we would label common sense reasoning, draws upon past experience arid underlying analogies. For instance, the master chess player is not $\mathbf{a}$ better deductive engine than his novice counterpart. Rather, as Chase and Simon [14] have shown, he commands a vast repertoire of chess-board patterns and associated sirategies that comprise his past experience. And, when encountering a new chessboard situation he uses the relevant pattarns (which may only partially match the current position) to index the appropriate knowledge. Mechanics problems in physics are often solved by creating a simple mental model .. an analog of the real situation .. that preserves the significant properties. The model, created on the basis of past experience solving similar problems, is then used to instantiate one or more weil known principles of physics in a familiar manner and thereby obtain a solution $[27,15,10]$.

People's well-developed ability to perform analogical reasoning is at least partly responsible for what we call "common-sense" reasoning. Roughly speaking, analogical reas ning is the process by which one recognizes that a new situation is similar to some previously ericountered situation, and uses the relevant prior knowledge to structure and enri vone's understanding of the new situation. We refer to metaphorical reasoning as that subset of canalogical reasoning in which the analogy is explicitly stated or otherwise made evident to the understander. For instance, comprehending "John 
is an encyclopedia" entails metaphorical reasoning since the analogy between John and an encyclopedia is explicitly suggested. However, constructing a novel analogy in order to explain some new situation is a different task which requires searching memory for a previously encountered similar situation. Both of these forms of inference may be labeled common.sense reasoning in so far as they require access to large amounts of past knowledge and reaching conclusions withnut benefit of formal deduction.

\section{Patterns of Metaphorical Inference}

A metaphor, simile or analogy can be said to consist of 3 parts: a target, a source and an analogical mapping. For example:

John was embarrassed. His face looked like a beet.

Here the target is "John's face" and the source is "a teet". The analogical mapping transmits information from the source to the target dnmain. In this case, the mapping relates the color of John's face to the color of a beet. Our use of the same terminology to describe metaphors, similes and analogies reflects our opinion that they are all merely different linguistic manifestations of the same underlying cognitive process: analogical reasoning. That is, they differ primarily in their form of presentation rather than in their internal structure. Consequently, although our choice of terminology may indicate that we are centrally concerned with the phenomenon of metaphor, we mean to include simile and analogy as well.

\subsection{The Balance Pri:uciple}

Consider a prevalent metaphor: reasoning about imponderable or abstract entities as though they were objects with a measurable weight. One of several reasoning patterns based on this simple metaphor is the balance principle. The physical analog of this reasoning pattern is a prototypical scale with two balanced plates. Large numbers of metaphors appeal to this simple device coupled with the processes of bringing the system into (and out (?) equilibrium. First, consider some examples of the basic metaphor, in which the relevant aspect of an abstract concept maps onto the weight ${ }^{2}$ of an unspecified physical object.

Arms control is a woighty issue.

The worries of a nation weigh neavily upon his shoulders.

The Argentine air force launched a massive attack on the British fleet. One frigate was heavily damaged, but only light casualties were suffered by British sailors. The Argentines payed a heavy toll in downed aircraft.

\footnotetext{
Moss is virtually synonymous with weight in naive reasoning.
} 
Not being in the mood for heavy drama, John went to a light comedy, which turned out to be a piece of meaningless fluff.

Pendergast was a real heavyweight in the 1920s Saini Louis political scene.

The crime weighed heavily upon his conscionce.

The weight of the evidence was overwhelming.

\subsection{The Physicai Metaphor Hypothesis}

Woight clearly represents different things in the various metaphors: the severity of a nation's problems, the number of attacking aircraft, the extent of physical damage, the emotional affect on audiences of theatrical productions, the amount of political muscle (to use another metaphor), the reaction to violated moral principles, and the degree to which evidence is found to be corvincing. In general, more is heavier; less is lighter. One may argue that since language is heavily endowed with words that describe weight, mass and other physical attributes (such as height and orientation $[2 \epsilon, 8])$, one borrows such words when discussing more abstract entities [1] for lack of alternate vocabulary. Whereas this argument is widely accepted, it falls far short of the conjecture we wish to make.

Physical metaphor hypothesis: Physical metaphors directly mirror the underlying interence processes. Inferences patterns valid for physical attributes are used via the analogical mapping to generate corresponding inferences in the target domain.

In order to illustrate the validity of this hypothesis, consider a common inference pattern based on the weight of physical mbjects: The inference pattern is the balance principle mentioned earlier as applied to a scale with two plates. The scale can be in balance or tipped towards either side, as a function of the relative weights of objects placed in the respective plates. Inference consists of placing objects in the scale and predicting the resultant situation .. no claim is made as to whether this process occurs in a propositional framework or as visual imagery. although we favor the former. How could such a simple inference pattern be useful? How could it apply to complex, non-physical domains? Consider the following examples of metaphorical communication based on this inference pattern:

The jury found the weight of the evidence favoring the defendant. His impeccable record weighed heavily in his favor, whereas the prosecution witness, being a confessed conman, carried little weight with the jury. On balance the state failed to amass sufficient evidence for a solid case.

The SS.20 missile lips the balance of power in favor of the Soviets. 
Both conservative and liberal arguments appeared to carpy equal weight with the Fresident, and his decision hung on the balence. Hewever, his long-standing opposition to abortion tipped the scale in favur of the consorvatives.

The Steelers were the heavy pre.game fevorites, but the Browns started plling up points and accumulated a massive half.time lead. In spite of a late rally, the Steelers dir not score heavily enough to pull the game out.

The job applicant's shyness weighed against her, but her excellent recommendations tipped the scales in her favor.

In each example above the same basic underlying inference pattern recurs, whether representing the outcome of a trial, statements of relative military power, decision-making processes, of the outcome of a sporting event. The inference pattern itself is quite simple: it takes as input signed quantities .. whose magnitudes are analogous to their stated "weight" and whose signs deperid on which side of a binary issue those weights correspond .. and selects the side with the maximal weight, computing some qualitative estimate of hoiv far out of balance the system is. Moreover, the inference pattern also serves to infer the rough weight of one side if the weight of the other side and the resultant talance state are known. (E.g., If Georgia wonn the lootball game scoring only 14 points, Alabama's scoring must have been really light)

Our point is that this very simple inference pattern accounts for large numbers of inferences in mundane human siiuations. Given the existence of such a simple and widely applicable pattern, why should one suppose that more complicated inference methods explain human reasoning more accurately? We believe that there exist a moderate number of general inference patterns such as the present one which together span a large fraction of mundane human reasoning situaticns. Moreover, the few other patterns we have found thus far are also rooted on simple physical principles or other directly experienced phenomena.

\section{The Role of Metaphor in Common-Sense Reasoning}

Recently one of us developed a model uf analogical problem solving $[12,11]$ basad on the principle that past experience in solving particular classes of problems should play a central role in solving new problems of a similar nature. At the risk of oversimplification, analogical problem solving can be summarized as a four-stage process:

1. Recalling one or more past problems that bear strong similarity to the new problem.

2. Constructing a mapping from the old problem solution process into a solution process for the new problem, exploiting known similarities in the two problem situations. 
3. Instantiating, refining and testing the potential soiution to the new problem.

4. Generalizing recurring solution patterns into reusable plans for common types of preblems.

The analogical problem solving model finessed most of the issuea in bullding a computationally elfective mechanism to recall similar problein solving episodes from memory, but suggested a model built siung the lines of Schank's MOPS $[34,28,25]$, utilizing relativo invariance messures $[8]$ as a memory organization principle.

Most of the "action" in the analogical problem solving model dealt with the issue of constructing a mapping that would transter, modify, and augment a solution frcm a similar past problem situation to satisfy the requirements of the new problem situation. Here, we propose that the role of metaphors is to capture and communicate mappings from well known experiential domains to new, less structured comains. These mappings often fail to provide deep insight into the target phenomenon they seek to explain, but do provide easily and quickly a shallow level of understanding sufficient for most common, everyday purposes. For instance, stating that the prosscutor's evidence was counterbalanced by the defendant's alibi gives us all a "feel" for the present state of the trial. But, consider a situation where you, the reader, must step in for the temporarily ill prosecuting atiorney. Suddenly, your understanding of the irial is woefully inadequate. Questions arise such as: "Just how dia the defense witness counter the prosecutions evidence? Did it undermine the credibility of our witnesses? Did it beef up the defendant's story? Did new svirdence surface? Does the case now hinge upon a possible breakdown in our chain of evidence?" (to use other metaphors). Deeper reasoning about a particular topic may well be metaphorically based, or more deductive in nature. Whether or not a particular metaphor provides more than a casual level of understanding depends on the validity of the source as a model, as well as ine structure of the mapping [20]. In the next section we will examine further the close relationship between mappings and metaphorical understanding.

\section{Metaphorical Inference and The Mapping Problem}

Metaphors, similes and analogies are more than clever ways of re-stating the obvious. They are extraordinarily concise devices by which a writer can convey rew information, simply by signaing his audience that information in the source dnmain is applicable to the target domain. Presumabiy the reader has a coherent body of knowledge about the source and can transfer and adapt this information to the target. This saves both the reader and the writer a good deal of time and cognitive effort. However, before the reader can initiate the transfer he must identify the correspondences between the two domains and establish exactly what information in the source is applicable to the target, sin je much of it is clearly lappropriate. This is the central issue in metaphor comprehension; 
we reler to it as the arialogical mapping problem.

One might suppose that the reader finds the analogical mapping simply by comparing the source and target domains for similarities. However, several resaarchers $[8,30]$ have demonstrated that this domain comparison moriel is in fact 100 simplistic. Although diecovering similarities is an important component of the comprehension process, other strategies must be used as well. Indeed, when learning new material. the reader knows little about the target, hence domain comparison is, by itsell, inacequate. Clearly, if the purpose of the metaphor is to transfer information to the target domain from the source domain, then this information does not already exist in the target domain, and hence it can play no part in the comparison process. Furthermore, when one considers requirements for computationai tractability, it becomes evident that there must be strategies to help focus the comparison and constrain the matching process. Focusing limits the complexity of the comparison process and reduces spurious matches between the two domains. ${ }^{3}$ Our purpose in this section is to expiore some cognitively plausible focusing strategies which might be used to facilitate the construction of analogical mappings.

\subsection{Knowledge Acquisition via Analogical Mappings}

The following example, found in a children's book, illustrates an explanation in which the reader (presumably a child) is expested to create an analogical mapping and transfer information across domains.

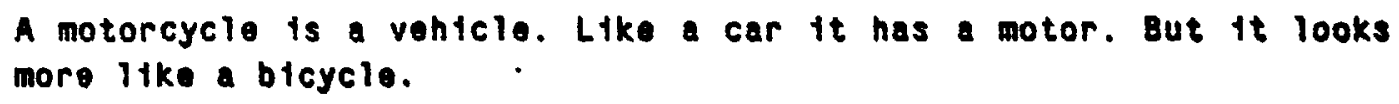

The author attempts to explain the concept of a motorcycle by referring to other, presumably more familiar, objects. But his statement implios much more than is explicitly stated. For instance, it suggests not only that a motorcycle has a motor, but that it has a motor in the same way that a car has a motor: that the motor is an internal combustion engine, it uses gasoline, it causes the machine to move, etc. The refaiance to a car is essential; consider the effect of substituting "electric shaver" for "car" in the example. (After all. electric shavers have motors too, but their motors are not a means of propulsion). Certainly. drawing an analogy to electric shave:s would not be nearly as helpful in communicating what a motorcycils is.

\footnotetext{
3implementing domain comparisons in a computer is typically accomplished by attempting to tind inatches between the representations of the target and source domains. As we shall see in the next section. these representatione are typically graphs or equivalent structures. Although the details of the matching process vary considerably depending on the representation system used, the computation can be quite expensive if pertormed upon arbitrary domains. Indeed. the related "Subgraph isomorphism" problem is NP.complete [19]. Given a precise formulation of the matching problem, it is ansy to demonatrate that it 100 is intractable unleas it is bounded in some principled way.
} 
Although analogies, such as the one above, can obviously be used to great advantage in transmitting new informatton, the reeder is ofton left in the position of not knowing how far carry the analogy. To a child who has never seen a motorcycle, the previous description of a motorcycle, though informative, is still quite ambiguous. "Doss a moiorcycle have pedals?" he may aek. In order to gauge the extent of the analogy, to writy which of his inference patterns relevant to cars and bicycies are valid for motoreycles, the child must either read turther or find a picture of a motorcyche. A priori, there is no way for him to be sure which interences to make. But at least the set of asnable questions he may ask will be focused by the analogy. Thus, it is ressonable to ack about handlebare or pedals, but not about whickers or winga.

In most mundane situations, knowing which inferences are correct saldom poses a significant problem for people, largely due to the fact that there are characteristic ways of expressing metaphors so that the mapping problem is easier to solve. For instance, consider that the truly novel metaphor is rarely encountered. Through frequent use, many metaphors acquire idiomatic meanings, to a greater or lesser degree. We refer to these metaphors as frozen. "John is a hog" and "Sheila is a dog" both exemplify frozen metaphors. The latter would probably be interpreted as a rude comment concerning Sheila's looks, rather than a compliment on her loyalty, which seems to be an equally reasonable interpretation given only one's knowledge about dogs. Frozen metaphors are easy to understand because the analogiral mapping has been (to some degree) precomputed, and so does not have to be reconstructed, only remembered and reapplied. Hence, neither a complex matching process nor prior knowledge about the target are necessary in order to find the mapping. There is little question of which are the right inferences and which are the wrong ones.

\subsection{Salience and Novel Melaphors}

If a metaphor is novel, other strategies are available for coping with the complexity of the mapping problem. One way is to focus on salient features of the source $[30,35]$. Consider the example "Billboards are like warts" in which both the target and source are familiar objects. Most people interpret this as meaning that billboards stick out, and are ugly. Their mapping relates attributes that are common to both source and target, but particula:ly emphasizes those such as "igliness" that are "high.salient" attributes of warts. the scurce. It is our contention that by focusing on prominent features and ignoring unimportant ones, the computational complexity of the mapping problem is reduced.

Concentrating the initial mapping to salient features of the source is an effective strategy even when one's knowledge of the target domain is limited. In fact, it is likely that the salient features are the very ones that should be mapped into the target domain, as is the case the following metaphor: 
RTO Ine. Is the Frodd te Laker of consuner electrontes.

Although the target is an unknown company, arid the metaphor is novel, it to understandable simply because the source, Freddie Laker of Laker Airines, has certain outstandingly salient featurea. Of course, the creator of the metaphor expects that his audience will all have the same opinion as to which of Laker's features are salient. Why cerrain features are considered universally salient whereas others are not is a difficult problem in its own right, one which will not pause to conalder hare.

We have examined wo types of metaphors which can be understoud in spite of incomplete knowledge of the target: frozen metaphors and motaphors based on the source's salient featurea. These illustrate just two of the many ways pragmatic considerations enable one to bypaes much of the complexity of the mapping problem. Occasionally, however, one cannot avoid more complex versions of the mapping problem. For us, this is the most interesting case. It occurs frequently during cxplanations involving extended analogies, such as when a grade-school mathematics racher begins his algebra ciass by proclaiming:

An oquation ts like a balance. You nust koop the sane anount of weight on each side of the equals sign....

Certainly there will be students in the class for whom this is a novel idea, and who spend the next 10 minutes desperately trying to find the intended analogical mapping. Or consider a secondary school biology text which begins a chapter on the human nervous systein by comparing it to a telephone network. Or a treatise on "liamlet" whose thesis is that the protagonist's life is a metaphor for adolescence. When confronted with one of these analogies in context, one may noed to search for appropriate hypotheses; one's analogical mapping will be elaborated and changed as one's understandini; of the target domain grows.

A good example of an extended analogy is provided by Milton Friedman in his Newsweek Column (of 12/27/82), as he attempts to explain recent fluctuations in the nation's money supply.

...Consider the record: $M_{1}$ grew at annual reates of 15.3 percent from October 1981 to January 1982; 1.2 percent from January 1982 to July 1982; 16.3 perceni from July 1982 to November 1982. Is it really conceivable that the fed produced these gyrations on purpose, given its repeated protestations that it was committed to a steady and moderate rate of monetary growth?

Why the gyrations? A better explanation is that the Fed is, as it were, driving a car with a highly defective steering gear. It is driving down a road with walls on toth sides. It can go down the middle of the road on the average only by first bouncing off one wall and then off the opposite wall. Not very good for the car or its passengers or bystanders, but one way to get down the road.

This interpretation raises two key questions: first, why doesn't the Fed replace the defective steering gear? Second, what course will this defective steering gear lead to over 
coming months...

This metaphor provides the reader with a clear, albeit simplistic, understanding of the situation without requiring much prerequisite knowledge about how the fed works. A possible remedy (replacing the steering wheel) is suggested based on inferences valid in the source domain. Most importantly, the passage implies that the Fed's control over the economy and where it is headed (one can hardly help but use metaphors here!) is not very accurate. Simply by invoking this metaphor Friedman communicates his belief that this situation is bad .. but not totally disastrous .. without ever having to explain the underlying monetary and fiscal reasons. In fact, when we informally questioned people about what exactly Friedman is referring to when he speaks of "walls", most admitted that they weren't really sure. A typical response was that the "walls" represented some sort of "limits". And yet, these people felt that they had understood, or gotten the gist of, the metaphor. Apparently one's analogical mapping does not have to be particularly detailed, as le ng as key inferences can be made. It seems that once certain connections or beachheads have been established between the target and source domains, people are content to incrementally elaborate the mapping as they find it necessary during further reading or problem solving.

\subsection{A Classification Based on Processing Requirements}

In our discussion thus far, we have identified various analogical mapping strategies whose applicability depends upon the properties of the metaphor under consideration. We therefore offer the following pragmatic classification, based on what we believe are meaningful distinctions in the type of processing employed during comprehension. We caution that these categories should not be viewed as distinct; it seems more reasonable to view metaphors as cccurring along a continuum with respect the criteria presented below.

- Frozen Metaphors :.. Example: "John is a hog." These have idiosyncratic, wellextablished meanings and therefore require little, if any, analysis or domain comparison during comprehension. Spurious inferences, such as John having a curly tail, snoutish nose or a tendency to crawl on all fours, do not typically enter the readers's mind.

- Partially Frozen Metapr: is .. Example: "The new evidence weighed heavily in the defendant's favor". The sance metaphor is a partially frozen metaphor; the details of the metaphor may vary from instance to instance, but the mappings remain fairly standard. Previous experience guides the mapping process, and thereby reduces the amount of domain comparison necessary to establish a new mapping.

- Novel One-shot Metaphors .. Example: "RTO electronics is the Freddie Laker of consumer electronics". These metaphors may require a considerable amount of computation in order to construct a satisfactory mapping. Various strategies, such as focusing on salient attributes, are employed both by the writer in creating the metaphor, and the reader in comprehending the metaphor. Typically these metaphors are used 
once, and then forgotten.

- Extenced Metaphors .. The quote from Friedman (above) is an example of an extended metaphcr. These are characterized by a relatively extensive mapping that is incrementally elaborated over time. The metaphor provides a model for reasoning about the target domain. Scientific analogies $[10,20]$ such as the nervous system/telephone network analogy fall into this category.

\section{Representing Metaphors: The LIKE Relation}

In the previous paragraphs we have discussed the problems involved in finding an analogicel mapping and making metaphorical inferences. We now turn our attention from the process of comprehension to issues of representation. How do we represent an analogical mapping in a computational model? We know that our representation must satisfy two requirements:

1. The representation must facilitate the transfer of information from the source domain to the target domain, and

2. it must be dynamic, enabling the analogy to be elaborated over time.

In this section we discuss how analogies (and metaphors) can be represented in semantic networks so as to satisfy these requirements. Although our work was motivated by representation languages such as KL.ONE [4], SRL [39], NETL [17] and KRL [2], we intend the ideas presented below to be applicable on a broad basis, and therefore make no commitment to any particular representational scheme. The notation presented in our diagrams is meant to be purely illustrativo.

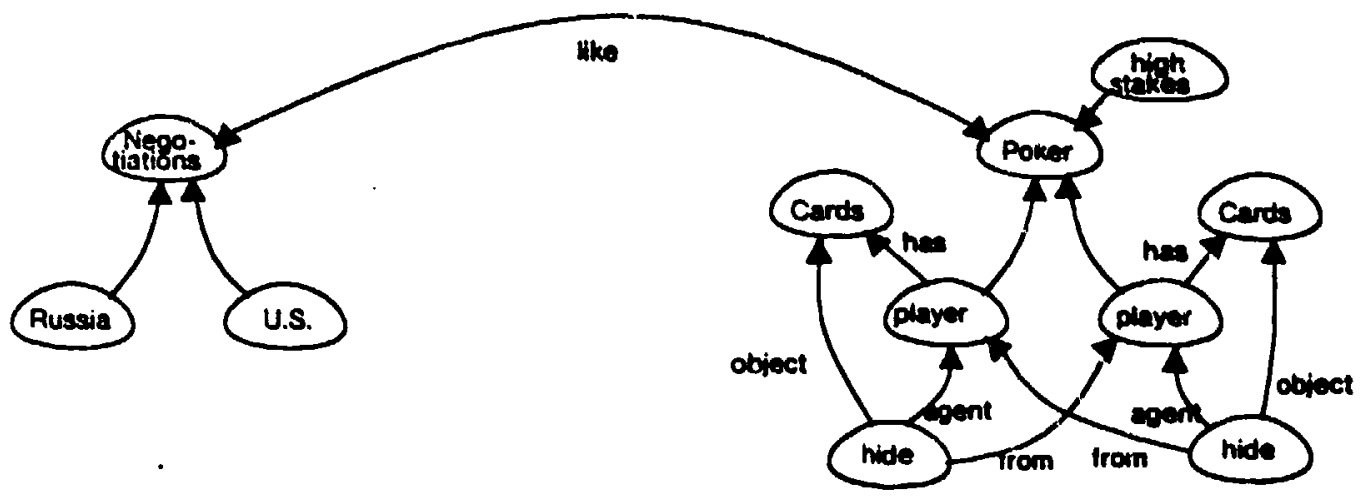

Figure 6-1: Using a single LIKE link to relate two domains

In a semantic network, knowledge ebout a domain is encoded as a graph. Typically the nodes 
represent concepts and the links relations. ${ }^{4}$ In order to represent an analogy weod a way of indicating that one domain is "like" another domain. For instance, we might want to represent the metaphor "The U.S./Russian arms negotiations is a high stakes poker game". Unfortunately, simply "linking" the target concept to the source concept with a "LIKE" relation 6-1 does not proviste us with the necessary functionality. We require a means of representing the various correapondences between the two graphs so that it is apparent how arms negotiations are like a game of poker. Russia and the U.S. are both being compared to poker players, each of which is withholding information from the other, etc. The representation must identily precisely which subconcepts and corresponding relations map into each other. A single link between two domains is just not expressive enough to represent mappings conveying our understanding of the motaphor.

We can attempt to remedy the situation by connecting all the corresponding subconcepts in the two domains with LIKE links. However, this multiple-link solution also falls since the lower-level links have meaning only in the context of the entire analogy. To see why, consider what might occur after representing and storing "The Democrats and the Republicans are like Coke and Pepsi", meaning that they are virtually indistinguishable except by self-chosen labels. One of the assertions contained in the semantic network would be "The Republicans are like Pepsi". In isclation, this fact is meaningless and potentially misleading. We must associate it with its analogical context. But, if only subconcept correspondences are stored, it is impossible to reconstruct the context because there is nothing in the knowledge base that represents the enalogy per se. The problem grows worse for more complex analogies because it is increasingly difficult to keep track of the various interrelationships between concepts given the growing forest of LIKE liriks. Essentially, the multiple-link solution is inadequate because it relies completely on a reductionistic representation for the analogy. (See Bobrow and Winugrad [2] and Schank [34] for discussions of more wholistic, reconstructive representations.)

In order to represent an arbitrary mapping between two domains, we propose to use a distinct entity, which we term a mapping structure. A mapping structure hunctions as a fiter, allowing explicitly specified types of information to be transierred from one domain to another. (In this reapect it plays a role similar to that of Winston's transfer frames $[38,37]$.) A mapping structure identilies the various correspondences in the source and target by providing a skeleton that specifies the analogical mapping in terms of common elements found in both domains. Figure $6-2$ is a schematic

\footnotetext{
4 Concepts may be decomposable, in whinh case a single node can be replaced by a network of lower. level nodes if desired. Exactly how this is managed is of importance to the domain matching process, but it need not concern us greathy for the purposes of this discusaion.
} 


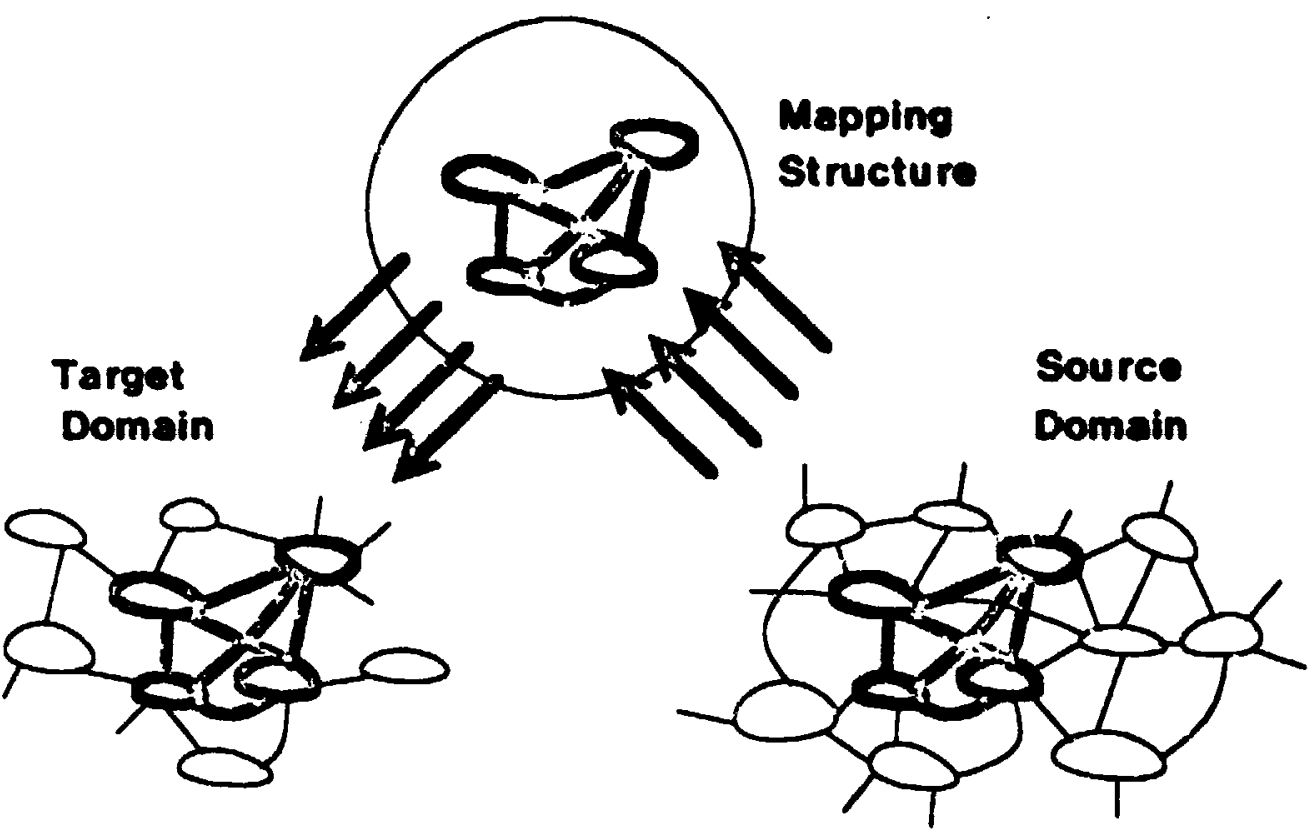

Figure 6-2: Schematic illustrating the role of mapping structures

which illustrates how the structure serves to coordinate the mapping by associating the two domains. The small graphs representing the target and source in the figure are mkant to reside withis much larger semantic networks.

In addition to the role they play in associating the source and target domains, mapping structures sorve to organize meta-information about the mapping. Inferences made as a result of the analogy are represented by collections of new nodes in the target domain. These new nodes are asociated with data-dependencins $[13,16]$ referring back to the mapping structure. (A data-dependency is essentially an indication of how the information was derived.) This enables the mapping structure to be extended incromentally while permitting subsequent verification or retraction of inferences. Because the mapping structure san te modified dynamically, at any particular time it represents the current conception of what the metaphor means. C course, this implies that the mapping structure must be retained for some unspecisied duration. We assume that the mapping structure will be "forootten" (i.e. discarded by some autonomous process supporting the representation syatem) if it dos not continue to be accessed as a source of inferences when target dornain information is retrioved from or added to memory.

Figure 6.3 shows a simplified representation for "Bulgaria is a Russian Puppet". The dotted lines around the CONTROL nocte in the target domain are meant to sulggest that this is an inference made 


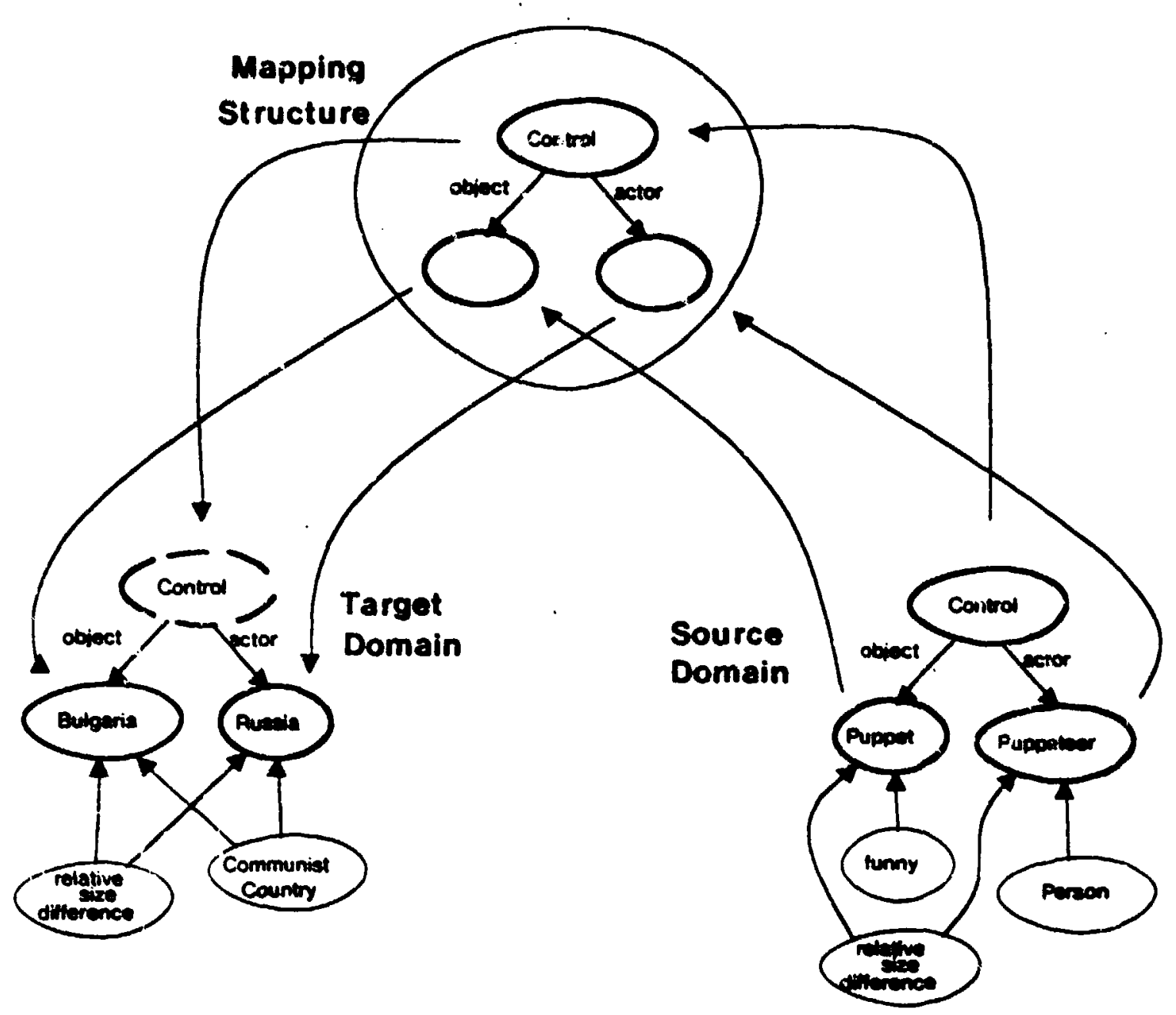

Figure 6.3: Simplificd representation of "Bulgaria is a Russian puppet".

as a result of the mstaphor. For simplicity, only the directly relevant sections of the target and source domains are actually shown. In addition, several nodes which do not take part in the mapping, such as the FUNNY attribute describing puppets, are included for illustrative purposes. Note that in both the target and the source, there is a node signilying the "relative size differences" of the objects (admittedly a gross representational simplification). Although this node is not part of the mapping, it might very well be included later if the mapping is extended.

As we pointed out in the previous section, a metaphor may become frozen through frequent use. An advar'age gained ty the use of inapping structures is that we can model computationally this "freezing" process quite naturally. When presented with a novel metaphor, there is no recourse but 
to construct a brand new mapping structure. Howewer, supposes a motaphor is encoumternd whowe source is similar to the source of a previousty undarstood metaphor. In this case we can wee the mupping structure built eartior as a bmplate to help build the new structure. This constrains the domain comparison process because the relevent foatures of the source concept ere identified by the pre-existing mapping structure. While one must still locate corresponding teatures in the new terget domain, this too may be done efficientty if the two targets are similar.

The statement "John eats like a pig" is a typical example of a frozen metaphor. Notice that it th understandable even though we are using "John" as a generic person. In our model, the mapping structure corresponding to "...eats like a pig" is eseociated with the section of the knowledoe network where information about pigs' eating habits is stored. Parsing "John eats the a pig" requires retrieving this mapping structure, noticing the exact correspondence between the source in the structure and the source in the rew methphor, and then instantiating the structure with "John" as the target domain. Instantiation is relatively easy to do, because the mapping structure spacifies which nodes map from the source [8]. Obviously we have glossed over many important problems in this description, such as how mapping structures can be retrieved given a source description, and whather a new physical copy of the mapping structure must be generated for each instantiation of a frozen metaphor. These questions are being studied at the present time.

\section{Generalizing Mapping Structures}

In the previous section, mapping structures were proposed as a means for representing arbitrary inter-domain correspondences. If is our intention that mapping structures be viewed as data. structures which implement LIKE relaticins. That is, a LIKE relation still exists between the source and target domain of an analogy, but it is too complex to be implemented with a simple link. Instead, a more elaborate mechanism is required to represent the internal structure of the malogical relationship. The indirect implementation of an analogical relationship as a data structure declaratively specifying the mapping process provides a necessary extra level of abstraction along its functional dimension. Thus, one can refer to the entire analogy as a unit, or one can access and elaborate the constituent parts of the mapping structure.

At the present time, we are considering other relations that may be better implemented by mapping structures rather than by simple links. Perhaps the most obvious candidate the IS.A relation, which provides a way to structure a knowledge network into a type hierarchy so that properties of a class representative can be mapped automatically to members of that class. We refer to this as vertical 
inheritance, because each concept inherits from those sove it in the type hierarchy. Hatorically, vertical inheritance has been used in knowledge representation systums to implement certain types of default rassoning. For example, knowing that Clyde is an etephant, and elephants have irunks, a syetem might use inheritance to infer that Cyde has a trunk."

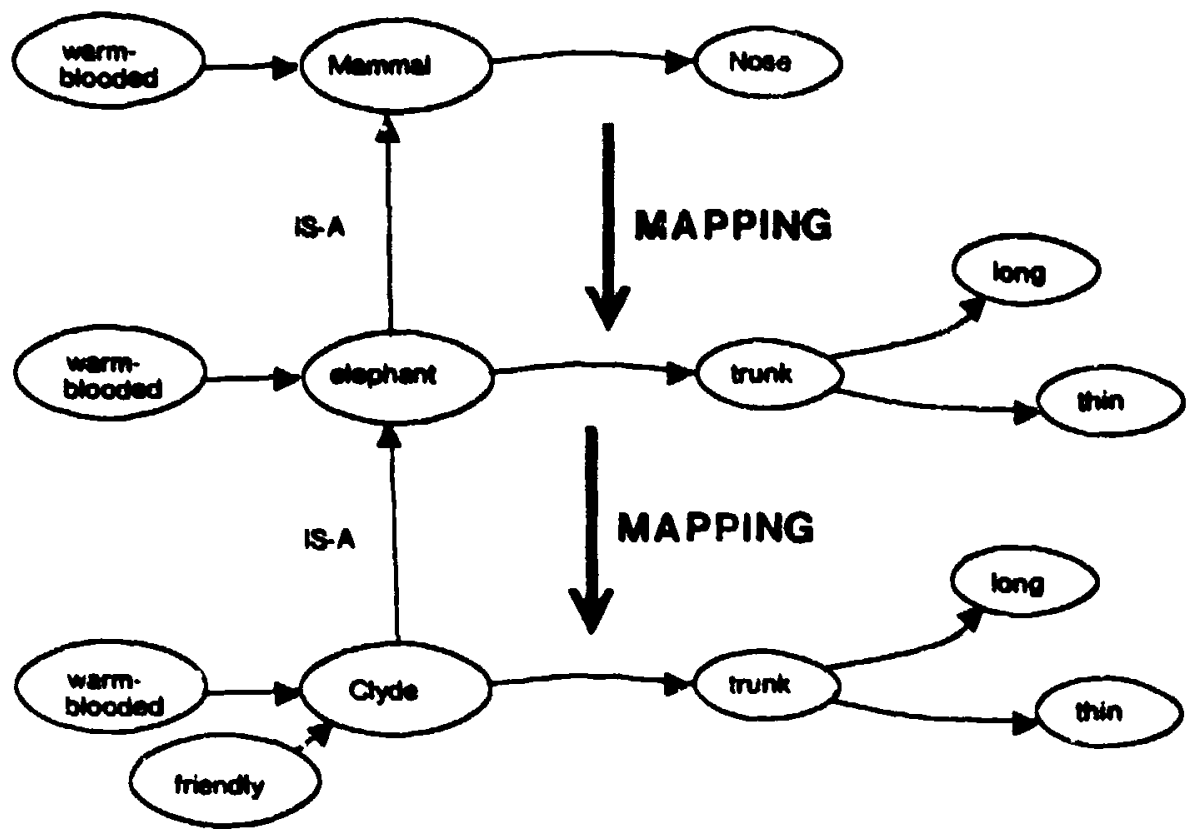

Figure 7.1: IS.A links and other inheritance relations entail mappings between concepts.

The IS.A relation requires that a mapping be established between the superordinate and subordinate concepts, so that properties of the superordinate can be inherited by the subordinate (Figure 7.1). Accordingly, it is natural to use a mapping structure to accomplish this as wes done for the LIKE relation. The mapping structure allows us to record what information may be transmilted from a particular superordinate, as well as to indicate explicitty what information should not be inherited. Although one assumes by default that all the information in the superordinate is shared by the subordinate, in many cases this can be contradicted. For example, if it is known that "Penguins do not fly", then the PENGUIN node should not inherit the FLIES attribute from BIRD. The mapping

\footnotetext{
In Al epplications, the type hierarchy bis elmoat never a strict hierarchy, but rather is a directed ecyclic graph or a tangiad hierarthy, in which a concept can have more than one superordinate. See Brachmen (3) for an overview of network-besed knowlader representation methode.

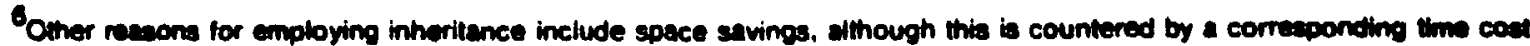
dunng retrieval operations. In addition, updating the knowledge base can be simpler; to add the fact that mammats ere marm blooded, only single node must be changed. that of "TYPICAL.MAMMAL", rather than having to find and change each and every node in the notwork denoting John, Mary, Folix the Cat, saber-10oth tigers, and all other mammab.
} 
structure provides us with a view of a penguin as a bird ${ }^{7}$ The key point we maan to empheaize ts that both the LIKE relation and the IS-A relation require mappings between concepts. This similarity has prompted some to describe the analogical mapping process as lateral inheritance, since information is mapped laterally between concepts in the type hierarchy. If one accepts this viempoint, thcil mapping structures begin to play a broed i ve in representing complex situationa.

\section{Tow ards a Computational Model of Metaphorical Inference}

The pervasiveness of metaphor in svery aspect of human communication has been convincingly demonstrated by Lakoff and Johnson [26], Ortony [31], Hobbs [23], and many others. However, with a fow exceptions $[23,8]$ the creation of a process model of motaphor comprehension and inference has not been of central concern. From a computational standpoint, metaphor has been viewed as an obstacle, to be tolerated at best and ignored at worst. For instance, Wilks [36] presents a few rules on how to relax semantic constraints in order for a purser to process a sentence in spite of tho metaphorical usage of a particular word. From our point of view, this attitude is not surprising, since few Al systems to date have used analogical ressoning as a primary inference method. Analogical reasoning has been viewed as a difficult problem in its own right, which must be solved before it can be incorporated in applications systems (such as parsers and medical diagnosis systems). Howewer, a robust system must be able to operate analogically, especially if intended for naive wers, otherwise they would find its lack of "common sense" intolerable. For example, a parser which could not understand metaphors. analogies, or similes would be usetul only in the most limited of situations." With these thoughts in mind we have begun initial work towards a purser which can racson metaphorically, and below present the following conceptual steps in the metaphor-recognition parsing process:

1. Identification of the source and target cuncepts. This is done during the parser's normal, non-metaphorical operation.

2. Recognition that the input currently being parsed cannot be handled literally, and is in fact an instance of a metaphor. This is actually a non-trivial task requiring considerable

\footnotetext{
7 Viess have played a central role in many knowhedge representation schemes. including those of and Moore and Newn [29]. Babrow and Winograd [2]. and Wright and Fox [30]. To indicate the noed tor more floxibte mappinos that anople all-or-nothing interitance, consides the rect that the average mammal may be 3 leet tall, or may range from $21 / 2$ ineh to 21 loet tali. Whereas we want our concept of "Girafte" to inherit most of our knowbodge of memmats. wn clearty do not wumt to say thes

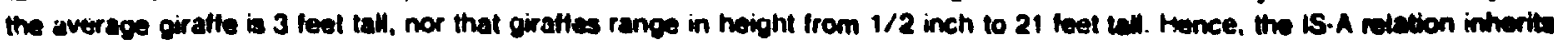
onty certain clasaes of attributes and excludes others; typically intrinsic properties of individual members are inherived wherees eagregate set properties we not. A mapping structure can be used to make explicit statementa such a the one sbowe. regarding the information that may be transmitted from one concept to enother via any particular inheritance lint (18, ef]

${ }^{8}$ Sheptics who dispute this claim are invited to examine any source of common weryday text. such as a copy of Thro magazime or even the New York Times Financial section, and count the number of metaphors occurring on a single pece.
} 
sophistication. For example, the parser must realize that the input is not simply crroneous. This judgement depends to a 'arge degree on pragmatic considerations.

3. Creation of an analogical mapping froin the source domain unto the target domain so that corresponding subconcepts in the two dornains map to each other. This phase may be broken doivn further as follows:

a. Search for a pre-existing mapping structure asscciated with the source domain.

b. If any such structure is found, check whether it is appropriate with respect to the new target donain. This is done by building incrementally a new mapping structure containing the same nodes as the old structure. As each new node is created a corresponding node in the target domain must be identified.

c. If no pre-existing mapuing structure is found for the source, or those that are found prove to be inappropriate :Jr the new target, then a new mapping must be constructed from scratch. A matching algorithm must search the two domains in order to find similarities. In this case, one should use as many heuristics as possible for reducing the amount of domain comparison that must be done. Possible heuristics include focusing on salient concepts in the source, and focusing on certain categories of knowledge which tend to be mapped invartant in meaningful metaphors [8].

4. Once corresponding nodes in the two domains have been identified (by constructing a mapping structure). knowledge from the source can be added to the mapping, thereby generating corresponding inferences within the target domain. ${ }^{2}$ In an abstract sense, this mechanisin accomplishes an implicit transfer of infonration from the source to the target. Verification that the metaphorical inferences are compatible with the target domain is an integral part of this process.

Whather or not it is possible to develop a rebust metaphor comprehension system with today's technology is a matter of debate. Metaphorical understanding requires a potentially vast amount of world knowledge, as well as an efficient way of comparing large domains for similarities. However, we feal that even a fragile, partial model built along these lines is a worthwhile endeavor, since eventually these problems must be solved in order to create a truly intelligent parser and inference system.

In cooperation with work towards a model of metaphor understanding, we are also studying the role that metaphorical inference plays in scientific reasoning. As discussed eartier, metaphorically-based

\footnotetext{
Qwe ecknowledge that the model as specified does not account for the way people understand metaphors such es "ieny is a block of ice", in which the properties trensterred from the source are themealves mataphnical. The matephor trenelers the property "cold" from ice to Mary. but this is a metaphor within a melaphor because we are refering to Mary's personality rather then her temperature. Metaphors occur in ell shapes and sizes. and we have not addreased many of the subtier nuances of the phenomenon in this paper. We do betieve, however, that the model can be elaborated to handle more sophiaticated metughors without reviaing the genoral fremework we have presented.
} 
general patterns of irference do nof appear confined to naive ressoning in mundane situstions. Gentner [20] and Johnson [24] have argued the significant role that metaphor plays in formulating scientific theories. In preliminary inverigations, Larkin and Carbonell $[27,10]$ have isolated general inference patterns in scientfic reasoning that transcers the traditional boundaries of a science. For instance, the notion of equilitrium (of forces on a rigid object, or of ion transfer in anueous solutions, etc.) is, in essence, a more precise and general formulation of the balance metaphor. Reasoning besed on recurring general inference patterns seems common to all espects of human cognition. These patterns encapsulate sets of rules to be used in unison, and thereby bypass some of the combinatorial search problems that plague more traditional rule-based deductive inference systems. The inference patterns are frozen froin experience and generalized to apply in many retevent domains.

At the present stage in the investigation, we are searching for general inference patterns and the metaphors that give rise to them, both in mundane and in scientific scenarios. As these patterns are discovered, they are cataloged ancording to the situational features that indicate their presence. The basic metaphor uriderlying each inference pattern is recorded along with exemplary linguistic manifesiations. The internal structure of the inference patterns themselves are relatively simple to encode in an Al system. The difficulty arises in connecting them to the external world (l.e., establishing apprupriate mappin? ?s) and in determining their condizions of applicability (which are more accurutely represented as partial matches of the situatinns where apply. :ather than as simple binary tests). For instence, it is difficult to formulate a general process capable of drawing the mapping between the "weight" of a hypothetical object and the correspending aspect of the non. physical eritity under r.onsideration, so that the balance inference pattern my apply. It is equally difficult to determine the degree to which this of any other inference pattern can make a useful contribution to novel situaticr.3 tha: vear sufticient similarity to past experience [12].

\section{Conclusions}

In this paper we hav3 analyzed the role of metaphors in common sense reasoning. In particular, we showed how the balance metaohor exemplifies metaphorical inference, suggested that inference patterns valid for physical domains might provide the foundation upon which much of human common-sense reasoning rests, and provided the first steps toward a computationally-effective method for representing analogical mappings. However, since the current study is only in its initial stages. the hypothesis that metaphorical inference dominates human cognition retains the status of a conjecturs, pending additional investigation. We would say that the weight of the evidence is as yet insufficient to tip the academic scales. 
Our investigations to date sugnest that intensitied efforts to resoive the questions raised in this paper may prove fruittul, in sodition to pursuing the following related recearch objectives:

- Develop an augmented representtation language that handlas anelogical mappings as a natural operation. We insend to start from a lairly flexible, operational languege auch a SRL [39]. Using this language wo intend to bulld alld test a system that acquiras new information from external me'apherical explanations.

- Continue to divelop the MULTIPAR multi-strategy parsing systion $[21,22]$ and incorporate within its evolving flexible parsing strategies a means of recognizing and processing metaphors along the lines mentioned in this paper.

- Examine the extent to which linguistic metaphors reftect undertying inference patterns. The existence of a number generally useful interence patterns based on undertying metaphors provides evidence against, but does not refute, the possibility that the vast majority of inetaphors remain mere linguistic devices, as previously thought. In easence, the existence of a phenomenen does not necessarily imply its univarsal presence. This is a mattar to be rasolved by more cumprehensive future investigation.

- Investigate the close connection between models of experiential learning and metaphorical inference. In lact, our earlier investigation of analogical reasoning patterns in learning problem solving strategies first suggested that the inference patterns that could be acquired from experience coincide with those underlying many common metaphors $[12,8]$.

- Exploit the human ability for experientially based metaphoricial reasoning in order to enhance the educational process. In fact, Sleeman and others have independently used the balance metaphor to help teach algebra to young or learning disabled children. Briefly, a scale is viewed as an equation, where the quantities on the right and left hand sides must balance. Algobraic manipulations correspond to adding or deleting equal amounts of weight from both sides of the scale, hence preserving balarice. First, the child is taught to use the scals with color-coded boxes or different (integral) weights. Then, the transfer to numbers in simple algebraic equations is performed. Preliminary results indicate that childreir learn faster and better when they are eble to use explicitly this general inference pattern. We foresee other applications of this and other metaphorical inference patterns in facilitating instruction of more abstract concepts. The teacher must make the mapping explicit to the student in dumains alien to his or her past experience. As discussed eartier, establishing and instantiating the appropriate mapping is also the most problematical phase from a computational standpoint, and therefore should correspond to the most Jifficult step in the learning process.

Clearty, the possible research directions suggested by our initial investigations far outstrip our resources to pursue them in parailel. Hence. we will focus first on the basic representation and parsing issues central to a computational model of metap. ıorical reasoning. 


\section{Bibliography}

1. Black, M., Models and Metapnors, Cornell University Prews, 1902.

2. Bobrow, D.G. and Winograd, T., "An Overview of KRL, a Knowledge Represantation Language," Cognitive Science, Vol. 1, No. 1, 1977, pp. 3.46.

3. Brachman, F.J., A Struclural Paradigm for Representing Knowledge. PhD discertation, Harvard University, 1977. Also BBN report \#3888

4. Brachmen, R.J., "On the Epistemological Status of Semantic Networka," in Associative Networks, N. V. Findler, sd., New York: Acedemic Presen 1979.

5. Burstein, M.H., "Concept Formation Through the Interuction of Multiple Modets," Preceedings of the Third Annual Conterence of the Cognitive Science Society, 1881.

6. Carbonell, J.G., "Delault Reasoning and Inheritance Mechanisms on Type Hierarchies," SIGART, SIGPLAN, SIGMOD Joint volume on Data Abstraction, 1980.

7. Carbonell, J. G., "A Computational Model of Problem Solving by Analogy," Proceedings of the Soventh Inrernational Joint Conference on Artificial Intelligence, August 1981, pp. 147.152.

8. Carbonell, J.G., "Motaphor: An Inescapable Phenomenon in Natural Language Comprehension," in Strategies for Natural Language Processing. W. Lehnent and M. Ringle, ods., New Jersey: Erlbaum, 1982, pp. 415.434.

9. Carbonell, J. G., "Invariance Hierarchies in Metaphor Interpretation," Proceedings of the Third Meeting of the Cognitive Science Society. August 1981, pp. 292.295.

10. Carbonell. J. G., Larkin, J. H. and Reif, F., "Towards a General Scientific Roasoning Engine," Proceedings of the Eighth international Joint Conference on Artificial Intelligence. 1983, (Submitted)

11. Carbonell, J. G., "Experiential Leaming in Analogical Problem Solving," Proceedings of the Second Meeting of the American Association for Artificial Intelligence. Pittsburgh, PA, 1982.

12. Carbonell, J.G., "Learning by Analogy: Formulating and Generalizing Plans from Past Experience," in Machine Learning. An Artilicial Intelligence Approach. R. S. Michalski, J. G. Carbonell and T. M. Mitchell, eds., Tioga Press, Palo Alto, CA, 1983.

13. Cha:niak, E., Riesbeck, C. and McDermott, D., Artificial Intelligence Programming. Lawrence Erlbaum Associates, 1980.

14. Chase, W. G. and Simon. H. A., "Perception in Chess," Cognitive Psychology. Vol. 4, 1974, pp. 55.81.

15. Clements. J., "Analogical Reasoning Patterns in Expert Problem Solving," Proceedings of the Fourth Annual Conterence of the Cognitive Science Society, 1982.

16. Doyle, J., "A Truth Maintenance System." Artificial Intelligence, Vol. 12, No. 3, 1979, pp. 231.272.

17. Fahlman, S.E., NETL: A System for Representing and Using Real World Knowledge, MIT Press, 1979.

18. Fox, M.S., "On Inheritance in Knowledge Representation," Proceedings of the Sixth International Joint Conference on Artificial Intelligence, 1979. pp. 282-284. 
19. Garey, M. and Johnson, D., Computers and intrectability, W. H. Freeman, San Franciaco, 1970.

20. Gentner, D., "The Structure of Analogical Models in Science," Tech. report 4451, Bolt Eoranek and Nowman, 1980.

21. Hayes, P. J., and Carbonell, J. G., "Multi.Strategy Ccnstruction-Specific Parsing for Floxible Data Bese Query and Update." Proceedings of tive Seventh International Joint Conterence on Artificial Intelligence, August 1981, Pp. 432.439.

22. Hayes, P. J. and Carbonell, J. G., "Multi-Strategy Parsing and it Role in Robust Man-Machine Communication," Tech. report CMU.CS-81.118, Carnegie-Mellon University, Computser Science Depertment, May 1981.

23. Hobbs, J. R., "Metaphor, Metaphor Schemate, and Selective Inference," Tech. report 204, SRI International, 1979.

24. Johnson, M., "Motaphorical Reasoning", Unpublished manuscript

25. Kolodner, J. L., Autrieval and Organizational Strategies in Conceptual Memory: A Computer Model, PhD dissertation, Yale University, Nov. 1980.

26. Lakoll, G. and Johnson, M., Metaphors We Live By, Chicago University Press, 1980.

27. Larkin, J. H. and Carbonell, J. G., "General Patterns of Scientific Inference: A Basis for Robust and Extensible Instructional Systems", Proposal to the Office of Naval Research

28. Lebowitz, M., Generalization and Memory in an Integrated Understanding System. PhD dissertation, Yale University, Oct. 1980.

29. Moore, J. and Newell, A., "How can MERLIN Understand?," in Knowiedge and Cognition, L. Gregg, ed., Hillsdale, NJ: Erlbaum Assoc., 1974, pp. 253-285.

30. Ortony, A., "The Role of Similarity in Similes and Metaphors," in Metaphor and Thought. A. Ortony, ed., Cambridge University Press, 1979.

31. Ortony, A. (Ed.), Metaphor and Thought. Cambridge University Press, 1979.

32. Schank, R.C., "Reminding and Memory Organization: An Introductior, to MOPS," Tech. report 170, Yale University Comp. Sci. Dept., 1979.

33. Schank, R. C., "Language and Memory," Cognitive Science, Vol. 4, No. 3, 1980, pp. 243-284.

34. Schank, R. C., Dynamic Memory, Cambridge University Press, 1982.

35. Searle, J.R., "Metaphor," in Metaphor and Thought. A. Ortony, ed., Cambridge University Press, 1979.

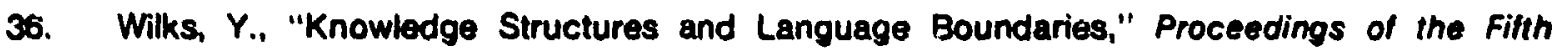
International Joint Conterence on Artiticial Intelligence, 1977, pp. 151-157.

37. Winston, P. H., "Learning and Reasoning by Analogy," Comm. ACM, Vol. 23, No. 12, 1979, pp. 689.703.

38. Winston, P.H., "Learning by Creating and Justifying Transfer Frames," Artificial Intelligence, Vol. 10, No. 2, 1978, pp. 147.172.

39. Wright, K. and Fox, M., "The SRL Users Manual," Tech. report, Robotics Institute, Carnegie. 
Mellon University, 1983.

t. 


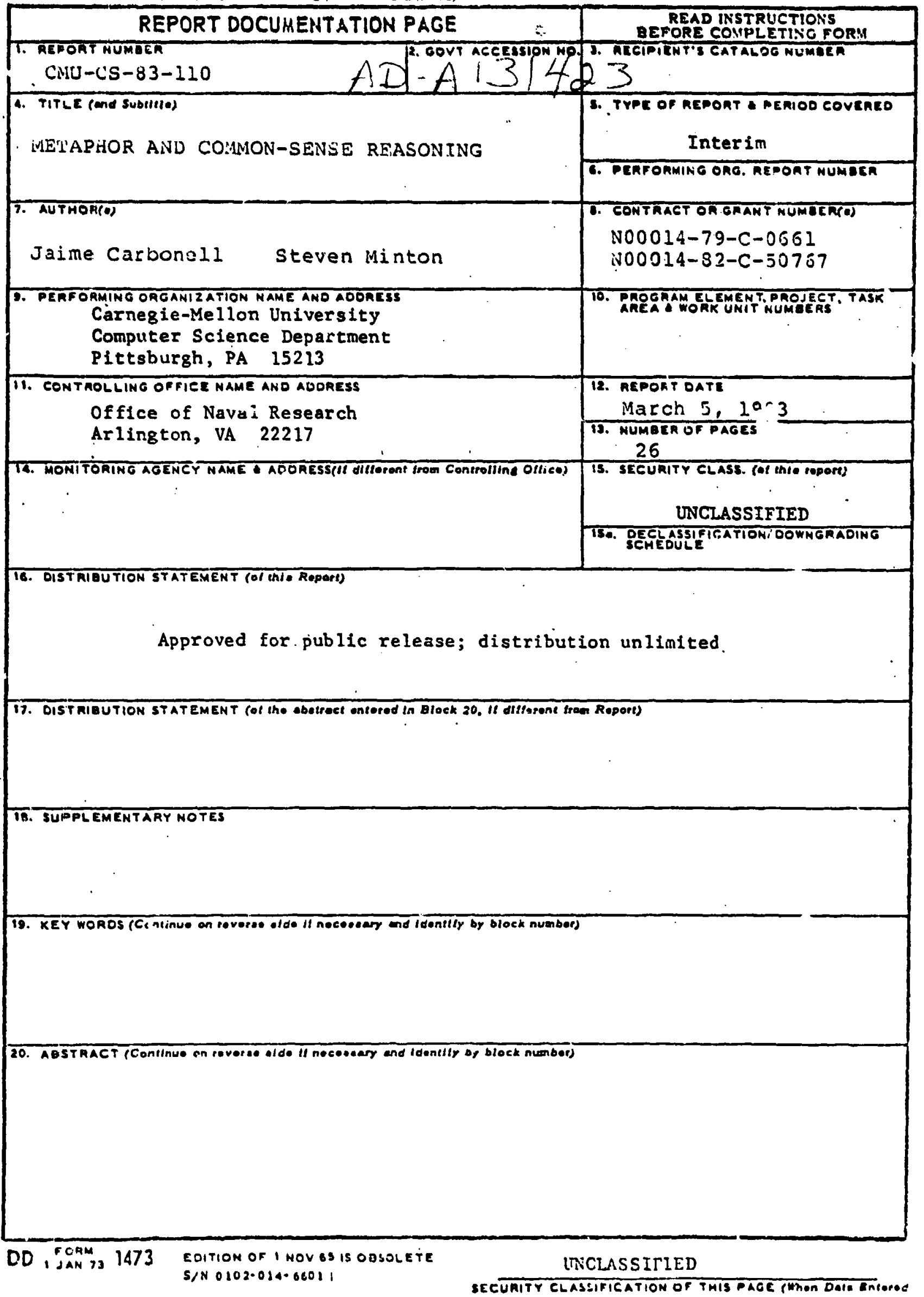

